\title{
SOBREVIVÊNCIA E CRESCIMENTO INICIAL DE ESPÉCIES ARBÓREAS NATIVAS NA RESTAURAÇÃO DE NASCENTES NO SUL DE MINAS GERAIS
}

\footnotetext{
Auwdréia Pereira Alvarenga (In memorian) ${ }^{1}$, Soraya Alvarenga Botelho ${ }^{2}$, André César Pinheiro ${ }^{3}$, Israel Marinho Pereira ${ }^{*}$

${ }^{1}$ Mestre em Ciências Florestais pela Universidade Federal de Lavras - UFLA

${ }^{2}$ Professora do Departamento de Ciências Florestais - UFLA, Caixa postal 37, CEP 37200-000, Lavras-MG

${ }^{3}$ Pós-Graduando do Programa de Pós-Graduação em Ciência Florestal do Departamento de Engenharia Florestal, Universidade Federal dos Vales do Jequitinhonha e Mucuri - UFVJM

${ }^{4}$ Professor do Departamento de Engenharia Florestal, Universidade Federal dos Vales do Jequitinhonha e Mucuri - UFVJM, Rodovia MGT 367 - Km 583, no 5000, Alto da Jacuba CEP 39100-00, Diamantina-MG ( ${ }^{*}$ imarinhopereira@gmail.com)

Recebido em: 08/04/2016 - Aprovado em: 30/05/2016 - Publicado em: 20/06/2016 DOI: 10.18677/Enciclopedia_Biosfera_2016_109
}

\section{RESUMO}

Este estudo teve como objetivo, avaliar a sobrevivência e o crescimento inicial de sete espécies arbóreas nativas na restauração ecológica de florestas ciliares no entorno de nascentes na região Sul de Minas Gerais. As espécies estudadas foram Trema micrantha (Cannabaceae), Guazuma ulmifolia (Malvaceae), Lithraea molleoides (Anacardiaceae), Cedrela fissilis (Meliaceae), Aspidosperma parvifolium (Apocynaceae), Machaerium villosum (Fabaceae) e Handroanthus serratifolius (Bignoniaceae). $O$ experimento foi realizado em delineamento inteiramente casualizado, em esquema fatorial $7 \times 3$ (espécies $\times$ sítios). Onze meses após 0 plantio verificou-se que, com exceção de $C$. fissilis e de $M$. villosum, todas as espécies apresentaram taxa média de sobrevivência igual ou superior a $80 \%$. A espécie que apresentou menor crescimento médio em altura após os onze meses foi C. Fissilis $(9,27 \mathrm{~cm})$. Com exceção dessa espécie, todas demonstram capacidade em se estabelecer neste tipo de ambiente e são recomendadas em projetos de restauração ecológica de florestas ciliares nessa região.

PALAVRAS-CHAVE: Enriquecimento de florestas tropicais, florestas ripárias, recuperação.

\section{SURVIVAL AND INITIAL GROWTH OF NATIVE TREE SPECIES IN THE RESTORATION OF SPRINGS IN THE SOUTH OF MINAS GERAIS}

\section{ABSTRACT}

This study aimed to quantify the survival and early growth of seven native tree species in ecological restoration of riparian forests in the vicinity of springs in the Southern region of Minas Gerais. The species used were Trema micrantha (Cannabaceae), Guazuma ulmifolia (Malvaceae), Lithraea molleoides (Anacardiaceae), Cedrela fissilis (Meliaceae), Aspidosperma parvifolium (Apocynaceae), Machaerium villosum (Fabaceae) and Handroanthus serratifolius (Bignoniaceae). The experiment was carried out in a completely randomized design, in a factorial $7 \times 3$ (species $\times$ locations). Eleven months after the plant it was found 
that, with the exception of $C$. fissilis and $M$. villosum, all species presented average rate of survival equal to or greater than $80 \%$. The species that presented the lowest average growth in height after eleven months was $C$. fissilis $(9.27 \mathrm{~cm})$. With the exception this species, all demonstrated the ability to establish themselves in this type of environment and are recommended in projects of ecological restoration of riparian forests in the region.

KEYWORDS: Enrichment of tropical forests, riparian forests, recovery.

\section{INTRODUÇÃO}

As florestas ciliares proporcionam grande importância na proteção dos cursos d'água, atuando como barreiras para a entrada de nutrientes, sedimentos, adubos e defensivos agrícolas para os rios, bem como o processo de erosão, o qual provoca assoreamento dos mananciais e altera as características físicas, químicas e biológicas dos corpos d'água, influenciando na quantidade e qualidade da água (ANDRADE et al., 2005; CASTRO et al., 2012). Estas florestas desempenham um papel muito importante na proteção e abrigo à fauna (corredores ecológicos), bem como a manutenção da biodiversidade e estabilização de encostas, entre outros e sua efetividade depende do estado de conservação e largura (CURY, 2011).

Existem leis brasileiras que visam à conservação dos recursos naturais e dentre elas, destaca-se o Código Florestal Brasileiro (Lei no 12.651/2012), o qual define e regulamenta as APP's (OKUYAMA et al., 2012). Mesmo sendo de fundamental importância ecológica e também protegida pela legislação, estas áreas ainda vêm sofrendo alterações em grande parte do território brasileiro. A falta de informação sobre os impactos ambientais causados pela retirada desta vegetação coloca em risco a sustentabilidade desses ecossistemas e dos recursos hídricos (IORI et al., 2012) e que, além disso, vem sofrendo pressão antrópica com as expansões agrícola, urbana e industrial (MELO et al., 2013; MARTINS, 2014). Portanto, torna-se necessário adotar práticas que visem à recomposição da vegetação no entorno das nascentes e ao longo dos cursos d'água.

Atualmente, devido ao avançado grau de perturbação que atinge grandes áreas de vegetação florestal, a restauração ecológica principalmente por meio do plantio de mudas dos diferentes grupos sucessionais tem sido o método mais utilizado em estudos de revegetação (RUIZ-JAEN \& AIDE, 2005; CASTRO et al., 2012). RUIZ-JAEN \& AIDE (2005) afirmam ainda que o uso dessa prática, bem como a descrição do sucesso nos estudos de restauração ocorre em maior número na América do Norte comparado ao resto do mundo e ressaltam que este fato não está atrelado ao maior de número de áreas degradadas nesta região e sim às leis ambientais destes países.

O uso inadequado dos recursos naturais vem se tornando cada vez mais objeto de estudo entre os pesquisadores, devido à situação atual de degradação dos ecossistemas, bem como a poluição da água. Gerando ainda, uma necessidade de se criar soluções que visem à conservação desses recursos essenciais à vida (FARIA et al., 2012; OLIVA JR. \& SOUSA, 2012; SOUZA et al., 2012; MARTELLI, 2013; FORTES et al., 2015; SILVA et al., 2015). Neste contexto, existe então a necessidade de mais estudos que subsidiem projetos de restauração florestal de forma técnica e científica, buscando subsidiar ainda a legislação. Além disso, estudos mais adaptados à realidade das florestas tropicais brasileiras se tornam imprescindíveis para o conhecimento silvicultural das espécies nativas.

Deste modo, objetivou-se avaliar a sobrevivência e o crescimento inicial de sete espécies arbóreas nativas em duas nascentes na região Sul de Minas Gerais. 


\section{MATERIAL E MÉTODOS}

O experimento foi implantado no entorno de duas nascentes na região sul de Minas Gerais, como parte do programa de recomposição de florestas ciliares no entorno de nascentes, rios e reservatórios.

As nascentes foram cercadas num raio de 50 metros e discriminadas em três sítios a partir de diferenças encontradas entre os ambientes, sendo: Nascente 1 (Sítio 1) - situada na Bacia do Rio Capivari, afluente do Rio Grande, margem

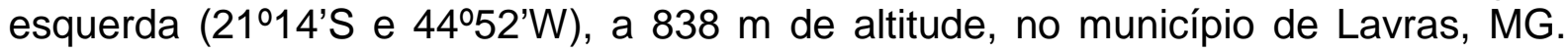
Este sítio não apresenta 50 metros de vegetação em seu entorno, com pouca presença de pastagem; Nascente 2 - situada na Bacia do Rio Capivari, afluente do

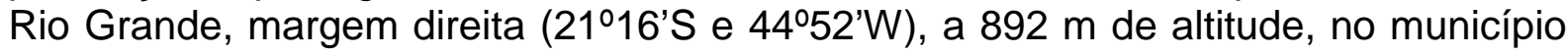
de Itumirim, MG, sendo Sítio 2: não possui a faixa mínima de 50 metros de vegetação no seu entorno, com presença de pastagem em início de regeneração natural e Sítio 3: área que apresenta pouca ou nenhuma vegetação colonizadora, além da presença de gado e locais com sulcos de erosão em diferentes profundidades. Todos os sítios estiveram ocupados, ao longo dos últimos anos, com pastagem utilizadas para pastoreio, até o isolamento de ambos os locais.

O clima da região é do tipo Cwa, segundo a classificação de Köppen, e a precipitação média anual é de $1.530 \mathrm{~mm}$ (SÁ JÚNIOR et al., 2012).

Para se obter as características químicas e granulométricas do solo, foram coletadas, em cada sítio, uma amostra composta de três amostras simples. O solo para análise foi retirado com auxílio de um trado holandês, a cerca de $20 \mathrm{~cm}$ de profundidade.

Utilizou-se como modelo de reflorestamento, o plantio misto entre espécies pioneiras e não pioneiras. Para tal, selecionou-se sete espécies arbóreas, sendo três pioneiras e quatro não pioneiras. A relação e classificação das espécies, quanto ao grupo ecológico, encontra-se na Tabela 1.

Para proceder com o experimento, realizou-se o plantio de mudas em janeiro com base no cultivo mínimo, utilizando como técnicas de preparo do solo, apenas a abertura de covas $(30 \times 30 \times 30 \mathrm{~cm})$, confeccionadas manualmente num espaçamento de $25 \mathrm{~m}^{2}(5 \times 5 \mathrm{~m})$, totalizando 35 covas por sítio e cinco covas para cada espécie. Utilizou-se na adubação de plantio, $150 \mathrm{~g}$ de superfosfato simples por cova. A altura média das mudas utilizadas no plantio foi de 23 e $17 \mathrm{~cm}$ para as espécies pioneiras e não pioneiras, respectivamente.

Foram realizadas capinas manuais com coroamento num raio de $50 \mathrm{~cm}$ ao redor das plantas. Antes do plantio, realizou-se o combate a formigas por meio do uso de termonebulizador. Durante os 11 meses de avaliação, utilizou-se iscas granuladas sempre que registrada a incidência de formigas nas áreas.

TABELA 1. Relação das sete espécies arbóreas utilizadas na restauração de florestas ciliares de duas nascentes na região Sul de Minas Gerais

\begin{tabular}{lll}
\hline Nome Científico & Família & GE \\
\hline Trema micrantha (L.) Blume & Cannabaceae & P \\
Guazuma ulmifolia Lam. & Malvaceae & P \\
Lithraea molleoides (Vell.) Engl. & Anacardiaceae & P \\
Cedrela fissilis Vell. & Meliaceae & NP \\
Aspidosperma parvifolium A.DC. & Apocynaceae & NP \\
Machaerium villosum Vogel & Fabaceae & NP \\
Handroanthus serratifolius (Vahl) S.Grose & Bignoniaceae & NP \\
\hline
\end{tabular}

Em que: $\mathrm{GE}=$ Grupo ecológico $(\mathrm{P}=$ Pioneira e NP = Não-Pioneira) (LORENZI, 1992; CARVALHO, 1994). 
As espécies foram selecionadas com base na sua grande ocorrência nos remanescentes da região e também pela disponibilidade de mudas. A distribuição das espécies foi feita em arranjo regular, alternando espécies pioneiras e nãopioneiras (1:1).

A avaliação do desempenho das espécies no campo iniciou-se quinze dias após o plantio e as avaliações seguintes foram realizadas mensalmente até dezembro (11 meses). Foram avaliados: a sobrevivência, pela porcentagem remanescente de mudas em cada leitura, em relação ao número inicial de mudas plantadas; a altura, medida entre a base do caule e a gema apical principal, utilizando vara graduada e o DAS (diâmetro do caule à altura do solo), utilizando paquímetro.

Utilizou-se um delineamento inteiramente casualizado (DIC), em esquema fatorial 7 (espécies) $\times 3$ (sítios), com cinco repetições, sendo cada parcela experimental constituída de uma planta de cada espécie. Os dados foram submetidos a análises de variância, em que se considerou as espécies em grupos individualizados de espécies pioneiras ( $T$. micrantha, G. ulmifolia e L. molleoides; e não-pioneiras ( $C$. fissilis, $A$. parvifolium, $M$. villosum e $H$. serratifolius.

Foi realizado, por meio do aplicativo Genes (CRUZ, 2001), um teste de normalidade de Lilliefors (CAMPOS, 1983) e para todos os sítios, os dados de altura das mudas foram transformados em $\sqrt{\mathrm{x}}$, e os dados de DAS em $\sqrt{\mathrm{x}+1}$. Foi realizada análise e as médias foram comparadas por meio do Teste de Scott-Knott $(p<0,05)$.

\section{RESULTADOS E DISCUSSÃO}

Os resultados da análise química e textural dos solos das duas nascentes estão apresentados na Tabela 2. Observou-se que entre as duas nascentes não houve diferenças em relação aos teores de nutrientes, ambas apresentando teores baixos de fósforo. Quanto à textura, o solo da nascente 1 foi classificado como textura média, já o da nascente 2 , enquadrou-se dentro da classe de solo muito argiloso no sítio 2 e como argiloso no sítio 3.

TABELA 2. Características químicas e granulométricas do solo de duas nascentes na região sul de Minas Gerais

\begin{tabular}{|c|c|c|c|c|c|c|c|}
\hline \multirow{3}{*}{ Elementos } & \multirow{3}{*}{ Unidade } & \multirow{2}{*}{\multicolumn{2}{|c|}{$\begin{array}{c}\text { Nascente } 1 \\
\mathrm{~S} 1\end{array}$}} & \multicolumn{4}{|c|}{ Nascente 2} \\
\hline & & & & \multicolumn{2}{|c|}{ S2 } & \multicolumn{2}{|c|}{ S3 } \\
\hline & & $\mathbf{R}$ & I & $\mathbf{R}$ & I & $\mathbf{R}$ & I \\
\hline pH em água $(1: 2,5)$ & & 5,8 & AM & 5,9 & AM & 6,1 & AF \\
\hline P (Fósforo Mehlich 1) & $\mathrm{mg} / \mathrm{dm}^{3}$ & 0,9 & $\mathrm{~B}$ & 1,2 & B & 0,9 & $\mathrm{~B}$ \\
\hline K (Potássio Mehlich 1) & $\mathrm{mg} / \mathrm{dm}^{3}$ & 52 & $M$ & 30 & $\mathrm{~B}$ & 30 & $\mathrm{~B}$ \\
\hline Ca (Cálcio) & $\mathrm{cmolc} / \mathrm{dm}^{3}$ & 2,8 & $M$ & 3,9 & $M$ & 6,2 & A \\
\hline Mg (Magnésio) & $\mathrm{cmolc} / \mathrm{dm}^{3}$ & 0,6 & $M$ & 1,5 & A & 2,8 & A \\
\hline Al (Alumínio) & $\mathrm{cmolc} / \mathrm{dm}^{3}$ & 0,2 & $\mathrm{~B}$ & 0,1 & B & 0,1 & $\mathrm{~B}$ \\
\hline $\mathrm{H}+\mathrm{Al}$ (Acidez potencial) & $\mathrm{cmolc} / \mathrm{dm}^{3}$ & 3,2 & $\mathrm{M}$ & 2,6 & $\mathrm{M}$ & 2,3 & $\mathrm{~B}$ \\
\hline SB (Soma de bases) & $\mathrm{cmolc} / \mathrm{dm}^{3}$ & 3,5 & $M$ & 5,5 & A & 9,1 & $A$ \\
\hline $\mathrm{t}$ (CTC efetiva) & $\mathrm{cmolc} / \mathrm{dm}^{3}$ & 3,7 & $M$ & 5,6 & $M$ & 9,2 & A \\
\hline $\mathrm{T}(\mathrm{CTC}$ a pH 7,0$)$ & $\mathrm{cmolc} / \mathrm{dm}^{3}$ & 6,7 & $M$ & 8,1 & $\mathrm{M}$ & 11,4 & A \\
\hline V (Saturação por bases) & $\%$ & 52,5 & $M$ & 67,8 & $\mathrm{M}$ & 79,8 & A \\
\hline m (Saturacão por alumímio) & $\%$ & 5 & $\mathrm{~B}$ & 2 & $\mathrm{~B}$ & 1 & B \\
\hline P-rem (Fósforo remanescente) & & 26.8 & & 16.9 & & 19.9 & \\
\hline
\end{tabular}




\begin{tabular}{|c|c|c|c|c|c|c|c|}
\hline \multirow{3}{*}{ Elementos } & \multirow{3}{*}{ Unidade } & \multirow{2}{*}{\multicolumn{2}{|c|}{$\frac{\text { Nascente } 1}{\mathrm{~S} 1}$}} & \multicolumn{4}{|c|}{ Nascente 2} \\
\hline & & & & & & & \\
\hline & & $\mathbf{R}$ & I & $\mathbf{R}$ & I & $\mathbf{R}$ & I \\
\hline Areia & dag $/ \mathrm{Kg}$ & 44 & & 14 & & 31 & \\
\hline Silte & $\mathrm{dag} / \mathrm{Kg}$ & 24 & & 16 & & 28 & \\
\hline Argila & $\mathrm{dag} / \mathrm{Kg}$ & 32 & & 70 & & 41 & \\
\hline Classe textural & & & TM & & MA & & AR \\
\hline
\end{tabular}

Em que: $\mathrm{S} 1$ = sítio 1, S2 = sítio 2 e S3 = sítio 3; $\mathrm{R}=$ Resultados e I = Interpretação; $\mathrm{AM}=$ Acidez média; $\mathrm{AF}=$ Acidez fraca; $\mathrm{A}=$ Alto; $\mathrm{M}=$ Médio e $\mathrm{B}=$ Baixo; $\mathrm{TM}=$ Textura média; $M A=$ Muito argilosa e AR = Argilosa .

Os valores de taxa de sobrevivência das espécies pioneiras, 11 meses após o plantio das mudas no campo, são apresentados na Tabela 3.

TABELA 3. Valores médios de sobrevivência, em percentagem, das espécies pioneiras utilizadas na restauração de florestas ciliares de duas nascentes na região sul de Minas Gerais aos 11 meses de idade

\begin{tabular}{|c|c|c|c|c|}
\hline \multirow{2}{*}{ Nome científico } & \multirow{2}{*}{$\begin{array}{c}\text { Nascente } 1 \\
\text { S1 }\end{array}$} & \multicolumn{2}{|c|}{ Nascente 2} & \multirow{2}{*}{ Média } \\
\hline & & S2 & S3 & \\
\hline olleoides & 100 & 100 & 60 & $86,67 \pm 23,09$ \\
\hline Guazuma ulmifolia & 100 & 100 & 100 & $100 \pm 0$ \\
\hline Trema micranta & 40 & 100 & 100 & $80 \pm 34,64$ \\
\hline Média & $80 \pm 34,64$ & $100 \pm 0$ & $86,67 \pm 23,09$ & \\
\hline
\end{tabular}

Em que: S1 = sítio 1, S2 = sítio 2 e S3 = sítio 3.

A maior sobrevivência aos 11 meses de idade foi registrada para G. ulmifolia, que apresentou $100 \%$ de sobrevivência nas duas nascentes avaliadas. Já LACERDA \& FIGUEIREDO (2009), estudando a germinação e sobrevivência desta espécie em uma restauração de floresta ciliar no estado do Maranhão, encontraram $0 \%$ de sobrevivência, sendo esta contraindicada para uso em reflorestamentos, neste caso. Quando se compara a sobrevivência das espécies nas duas nascentes, verificou-se que o maior índice de sobrevivência foi encontrado para o sítio 2 (100\%), enquanto que o sítio 1 e o sítio 3 apresentaram sobrevivência semelhante.

As mudas de T. micrantha apresentaram 100\% de sobrevivência nos sítios 2 e 3, e decrescendo para $40 \%$ no sítio 1. MORAES et al. (2006) encontraram taxas de sobrevivência variando entre 97,1 e $61,4 \%$, aos 9 e 12 meses após o plantio. De acordo com estes mesmos autores, esta espécie geralmente apresenta mortalidade baixa e elevado crescimento inicial. Essas características, bem como o fato dos frutos de T. micrantha servirem como alimento para peixes (SOUZA \& MONTEIRO, 2005), torna esta espécie indicada para recompor ambientes ciliares. Sabe-se ainda, que a porcentagem de sobrevivência depende de fatores genéticos, da qualidade das mudas, do local de plantio e do clima, além da época de plantio. E considerando as condições deste estudo, somente as características de solo do local são diferentes.

Verificou-se para L. molleoides, $100 \%$ de sobrevivência no sítio 1 e no sítio 2. Já no sítio 3 , esta espécie apresentou $60 \%$ de sobrevivência. Este resultado evidencia a facilidade desta espécie em se estabelecer em ambientes perturbados. Os resultados do teste de médias para o crescimento em altura e DAS das espécies pioneiras, estão apresentados nas Tabelas 4 e 5. 
TABELA 4. Valores médios de altura $(\mathrm{cm})$ para as espécies pioneiras, aos 11 meses de idade em duas nascentes na região sul de Minas Gerais

\begin{tabular}{|c|c|c|c|c|}
\hline \multirow{2}{*}{ Nome científico } & \multirow{2}{*}{$\begin{array}{c}\text { Nascente } 1 \\
\text { S1 }\end{array}$} & \multicolumn{2}{|c|}{ Nascente 2} & \multirow{2}{*}{ Média } \\
\hline & & $\mathbf{S 2}$ & S3 & \\
\hline Lithraea molleoides & 38,00 a $A$ & 51,40 a $A$ & 33,00 a $A$ & $40,80 \mathrm{a}$ \\
\hline Guazuma ulmifolia & 22,70 a $A$ & 35,20 a $A$ & 44,40 a $A$ & $34,10 \mathrm{a}$ \\
\hline Trema micranta & 12,20 b B & 48,20 a $A$ & 35,60 a $A$ & $32,00 \mathrm{a}$ \\
\hline Média & $24,30 \mathrm{~B}$ & $44,93 \mathrm{~A}$ & $37,67 \mathrm{~A}$ & \\
\hline
\end{tabular}

Em que: $S 1$ = sítio $1, S 2$ = sítio 2 e S3 = sítio 3 . Médias seguidas de letras minúsculas iguais nas colunas ou maiúsculas nas linhas, não diferem entre si pelo teste de Scott-Knott a 5\% de significância.

TABELA 5. Valores médios dos diâmetros $(\mathrm{mm})$ para as espécies pioneiras, aos 11 meses de idade em duas nascentes na região sul de Minas Gerais

\begin{tabular}{|c|c|c|c|c|}
\hline \multirow{2}{*}{ Nome científico } & \multirow{2}{*}{$\begin{array}{c}\text { Nascente } 1 \\
\text { S1 }\end{array}$} & \multicolumn{2}{|c|}{ Nascente 2} & \multirow{2}{*}{ Média } \\
\hline & & S2 & S3 & \\
\hline Lithraea molleoides & 6,60 a $A$ & 7,80 a $A$ & 5,20 a A & $6,50 \mathrm{a}$ \\
\hline Guazuma ulmifolia & 5,00 a $A$ & 4,90 a $\mathrm{A}$ & 6,50 a $A$ & $5,50 a$ \\
\hline Trema micranta & $2,60 \mathrm{~b} \mathrm{~B}$ & 8,60 a $A$ & 8,80 a $A$ & $6,70 \mathrm{a}$ \\
\hline Média & $4,70 \mathrm{~B}$ & $7,10 \mathrm{~A}$ & $6,80 \mathrm{~A}$ & \\
\hline
\end{tabular}

Em que: $S 1$ = sítio $1, S 2$ = sítio 2 e S3 = sítio 3 . Médias seguidas de letras minúsculas iguais nas colunas ou maiúsculas nas linhas, não diferem entre si pelo teste de Scott-Knott a 5\% de significância.

Observando-se os valores de altura e DAS aos 11 meses, verificou-se que o comportamento dos dois estimadores foi semelhante. O crescimento médio foi maior nos sítios 2 e 3 e o crescimento entre as espécies não apresentou diferenças nestes dois locais. Para todas as espécies pioneiras avaliadas, os menores valores médios encontrados em altura foram no sítio 1. Este resultado pode ser considerado atípico, atribuído às características do solo, uma vez que neste sítio foi observado valores superiores para P-rem e K no solo (Tabela 2).

$\mathrm{Na}$ Tabela 6 são apresentados os índices de sobrevivência das espécies nãopioneiras aos 11 meses. Observou-se que $A$. parvifolium (100\%) e $H$. serratifolius $(93,33 \%)$ apresentaram as maiores taxas de sobrevivência média. Por outro lado, $C$. fissilis $(33,33 \%)$ foi a espécie com menor índice de sobrevivência média após 11 meses. SACRAMENTO et al. (2012), estudando o desenvolvimento inicial de quatro espécies lenhosas no estado da Bahia, constataram $57,2 \%$ e $62,6 \%$ de mortalidade para a espécie $C$. fissilis em áreas degradadas aos 12 e 24 meses, respectivamente, demonstrando sua sensibilidade a estes ambientes.

TABELA 6. Valores médios de porcentagem de sobrevivência das espécies não pioneiras, aos 11 meses de idade em duas nascentes na região sul de Minas Gerais

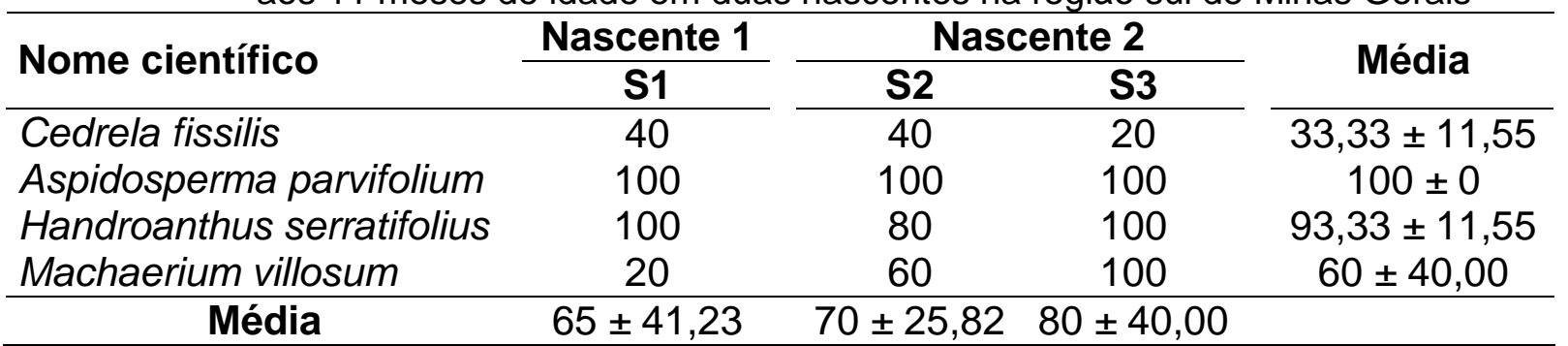

Em que: $\mathrm{S} 1$ = sítio $1, \mathrm{~S} 2$ = sítio 2 e S3 = sítio 3 . 
Os sítios 3 e 2 apresentaram as maiores médias de sobrevivência de espécies não-pioneiras, com $80 \%$ e $70 \%$ de sobrevivência, respectivamente. O sítio 1 apresentou a menor sobrevivência média das espécies aos 11 meses de idade, com $65 \%$. Estes resultados seguiram a mesmo padrão para as espécies pioneiras, fato provavelmente relacionado à sensibilidade das espécies quanto à tipologia das nascentes, independentemente do grupo ecológico.

No sítio 1 e no sítio 2, $A$. parvifolium e $H$. serratifolius apresentaram as maiores taxas de sobrevivência. Nestes mesmos locais, observou-se que as menores porcentagens de sobrevivência foram para $C$. fissilis e $M$. villosum. Já para o sítio 3, verificou-se $100 \%$ de sobrevivência para $A$. parvifolium, $H$. serratifolius e $M$. villosum. Neste sítio, $C$. fissilis apresentou a menor taxa média de sobrevivência (20\%).

$\mathrm{Na}$ Tabela 7 observa-se que houve diferença no crescimento em altura das espécies não-pioneiras após 11 meses. No entanto, não houve tal efeito em relação ao diâmetro (Tabela 8).

TABELA 7. Valores médios de altura $(\mathrm{cm})$ para as espécies não pioneiras, aos 11 meses de idade em duas nascentes na região sul de Minas Gerais

\begin{tabular}{|c|c|c|c|c|}
\hline \multirow{2}{*}{ Nome científico } & \multirow{2}{*}{$\begin{array}{c}\text { Nascente } 1 \\
\mathrm{~S} 1\end{array}$} & \multicolumn{2}{|c|}{ Nascente 2} & \multirow{2}{*}{ Média } \\
\hline & & S2 & S3 & \\
\hline Cedrela fissilis & 8,80 & 13,00 & 6,00 & $9,27 d$ \\
\hline Aspidosperma parvifolium & 32,00 & 34,40 & 27,80 & $31,40 \mathrm{a}$ \\
\hline Handroanthus serratifolius & 16,50 & 16,60 & 19,60 & $17,57 \mathrm{c}$ \\
\hline Machaerium villosum & 6,00 & 21,40 & 28,00 & $18,47 b$ \\
\hline Média & $15,82 \mathrm{~A}$ & $21,35 \mathrm{~A}$ & $20,35 \mathrm{~A}$ & \\
\hline
\end{tabular}

Em que: S1 = sítio 1, S2 = sítio 2 e S3 = sítio 3. Médias seguidas de mesma letra maiúscula nas colunas e minúscula nas linhas, não diferem entre si pelo teste de Scott-Knott a 5\% de significância.

TABELA 8. Valores médios de diâmetro $(\mathrm{mm})$, para as espécies não pioneiras, aos 11 meses de idade em duas nascentes na região sul de Minas Gerais

\begin{tabular}{|c|c|c|c|c|}
\hline \multirow{2}{*}{ Nome científico } & \multirow{2}{*}{$\begin{array}{c}\text { Nascente } 1 \\
\mathrm{~S} 1\end{array}$} & \multicolumn{2}{|c|}{ Nascente 2} & \multirow[t]{2}{*}{ Média } \\
\hline & & S2 & S3 & \\
\hline Cedrela fissilis & 3,00 & 3,30 & 2,00 & $2,80 \mathrm{a}$ \\
\hline Aspidosperma parvifolium & 5,90 & 5,00 & 4,80 & $5,20 \mathrm{a}$ \\
\hline Handroanthus serratifolius & 5,90 & 3,00 & 4,50 & $4,50 \mathrm{a}$ \\
\hline Machaerium villosum & 1,40 & 4,00 & 5,70 & $3,70 \mathrm{a}$ \\
\hline Média & $4,00 \mathrm{~A}$ & $3,80 \mathrm{~A}$ & $4,20 \mathrm{~A}$ & \\
\hline
\end{tabular}

Em que: $\mathrm{S} 1$ = sítio $1, \mathrm{~S} 2$ = sítio 2 e S3 = sítio 3 . Médias seguidas de mesma letra maiúscula nas colunas e minúscula nas linhas, não diferem entre si pelo teste de Scott-Knott a $5 \%$ de significância.

Dentre as espécies não-pioneiras, $A$. parvifolium foi a que mais se destacou no crescimento em altura, com média de $31,40 \mathrm{~cm}$ aos 11 meses de idade. A espécie $C$. fissilis apresentou o menor crescimento médio em altura aos 11 meses (Tabela 8). Este resultado assemelha-se ao obtido por SACRAMENTO et al. (2012), que registraram para $C$. fissilis o menor desenvolvimento inicial dentre as espécies avaliadas na recuperação de uma área degradada. Apesar de ser considerada secundaria tardia na posição sucessional (AGUIAR et al., 2001), essa espécie é frequentemente encontrada em clareiras e bordas de florestas (ANGELI, 2015), 
ambientes semelhantes às duas nascentes estudadas em relação à intensidade de luz. No entanto, DUZ et al. (2004) encontraram maior produtividade para esta espécie em ambientes com menor intensidade de luz, o que explica o baixo crescimento inicial de $C$. fissilis neste estudo.

A partir dos dados obtidos nas avaliações mensais, não foi possível o ajuste de equações de crescimento, em função do curto período avaliado e do pequeno crescimento neste intervalo. As Figuras 1 e 2 representam o crescimento médio em altura e DAS das espécies pioneiras e não-pioneiras em função da idade.

(A)
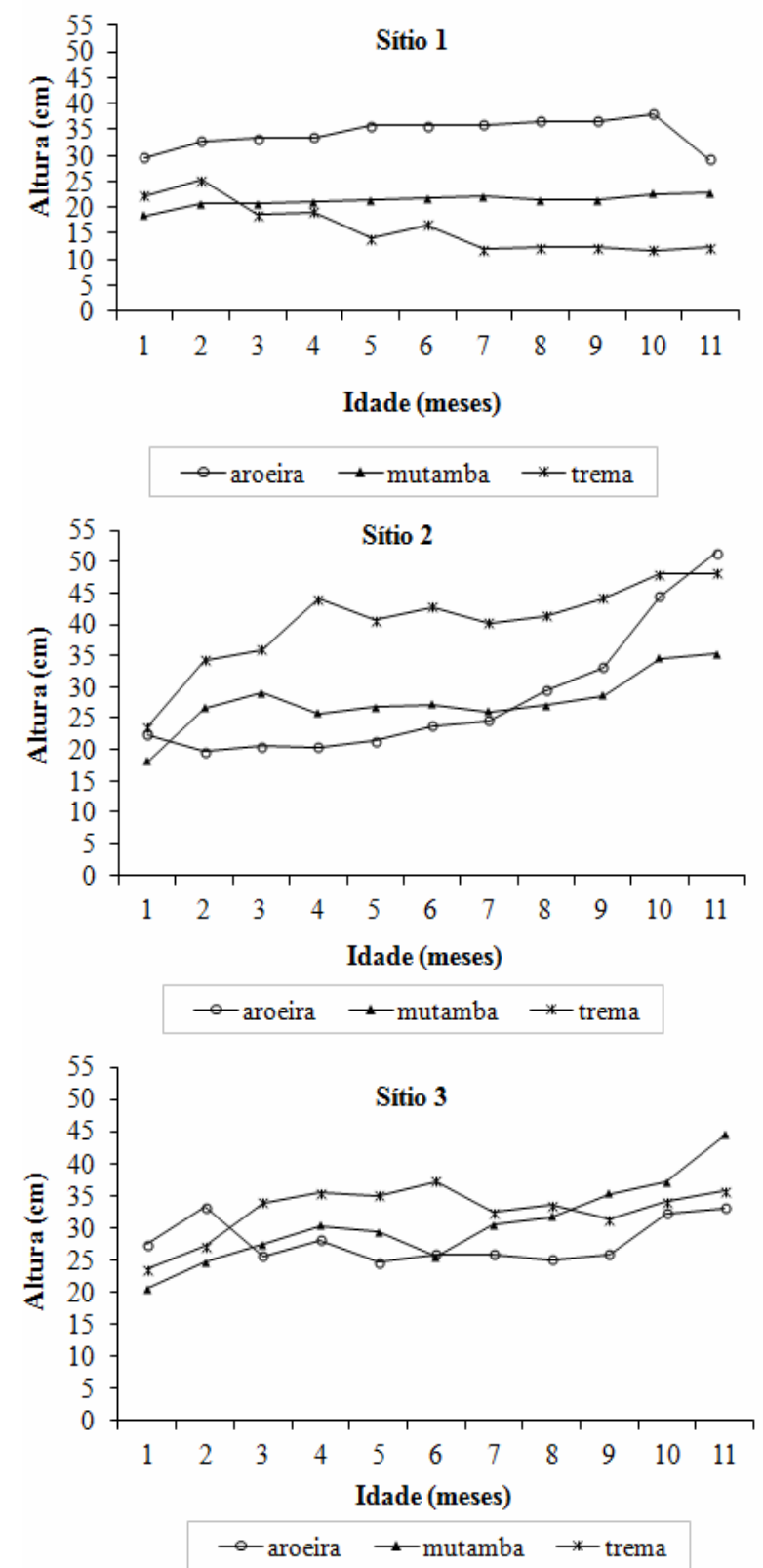

(B)
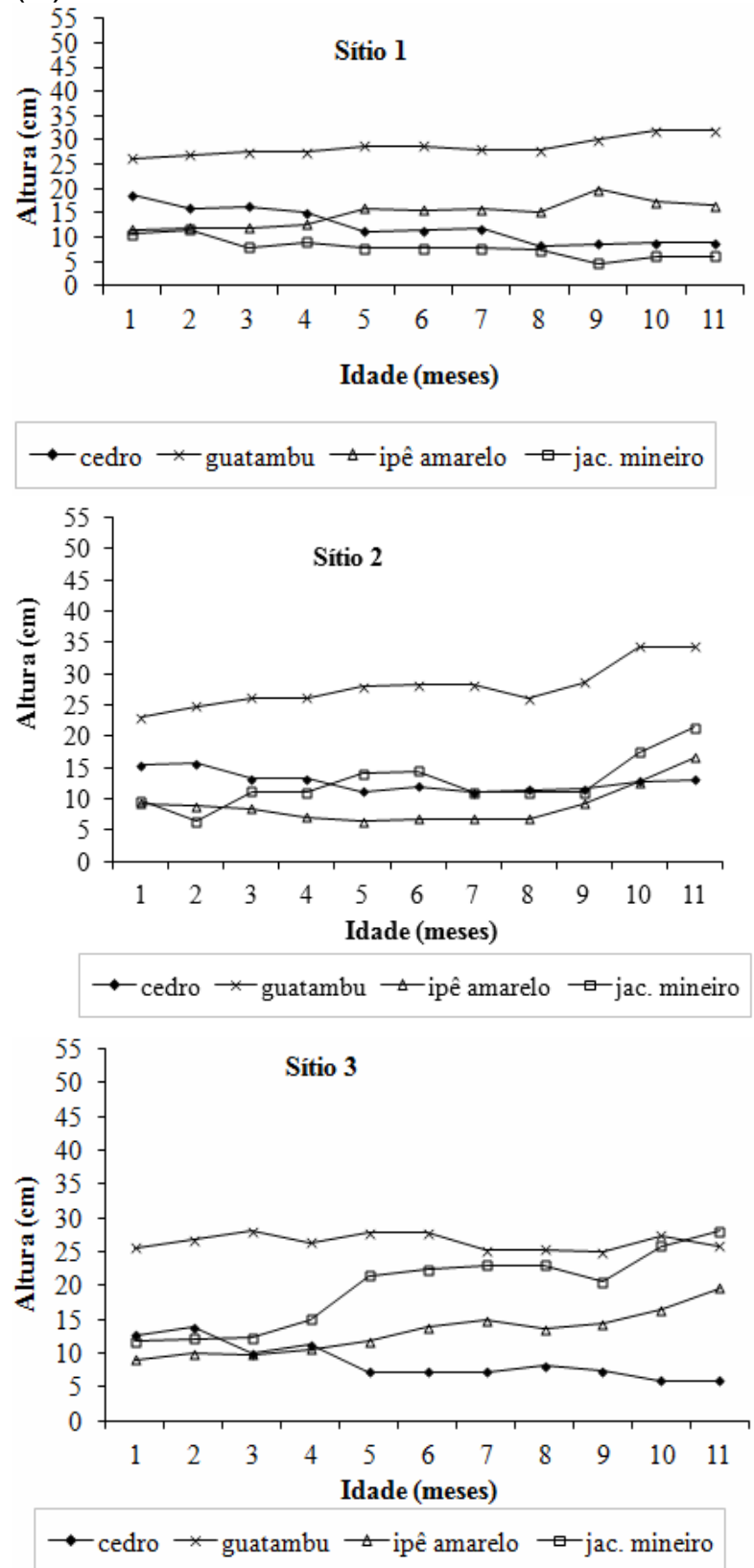

FIGURA 1. Curvas do crescimento médio em altura das espécies pioneiras $(A)$ e não pioneiras (B), no sítio 1, sítio 2 e sítio 3, até 11 meses de idade. Em que: Aroeira $=L$. molleoides; Mutamba $=$ G. ulmifolia; Trema $=T$. micranta; Cedro $=$ C. fissilis; Guatambu $=A$. parvifolium; $H$. serratifolius $=$ Ipê Amarelo e Jac. Mineiro $=M$. villosum. 
(A)
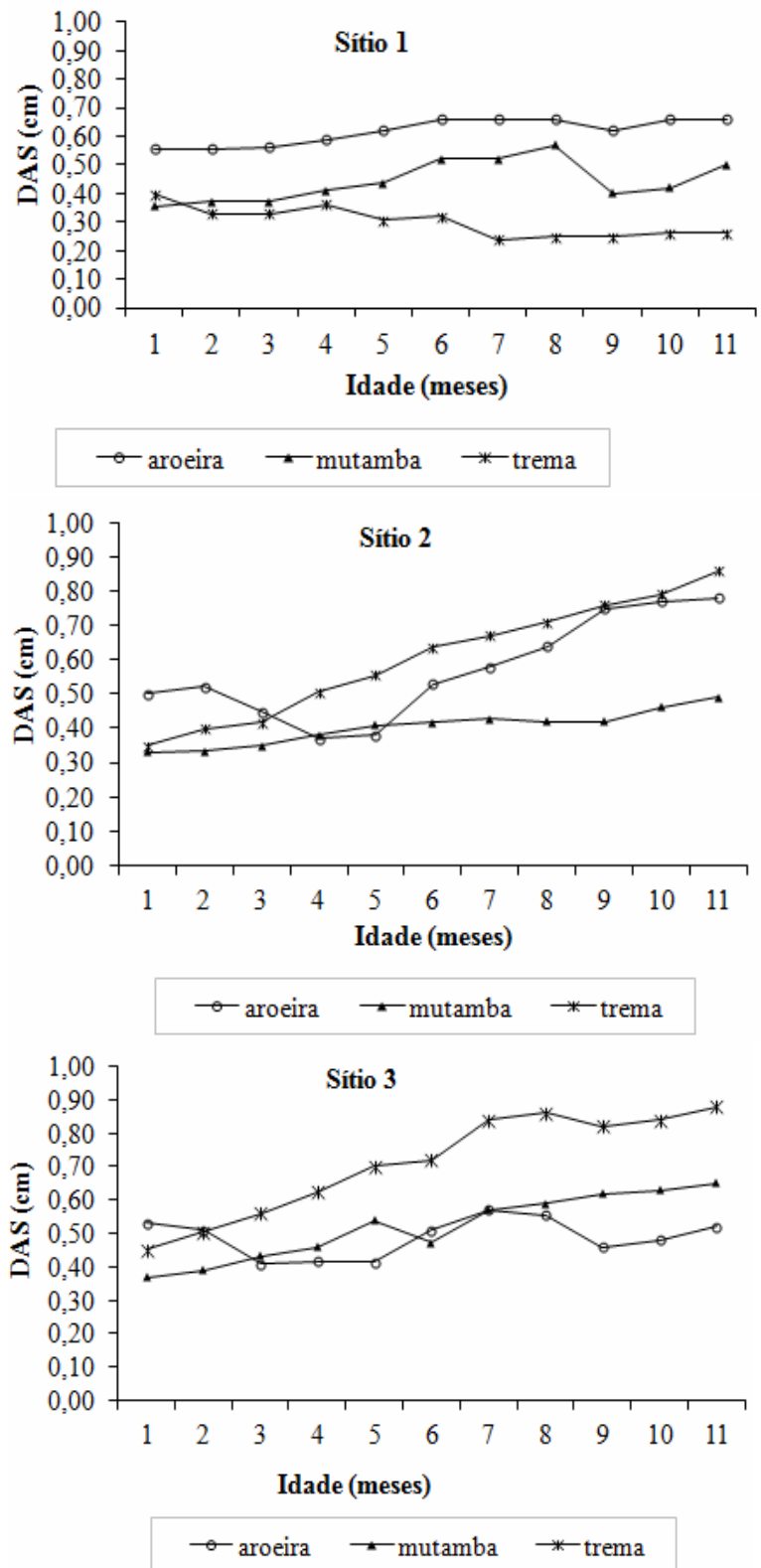

(B)
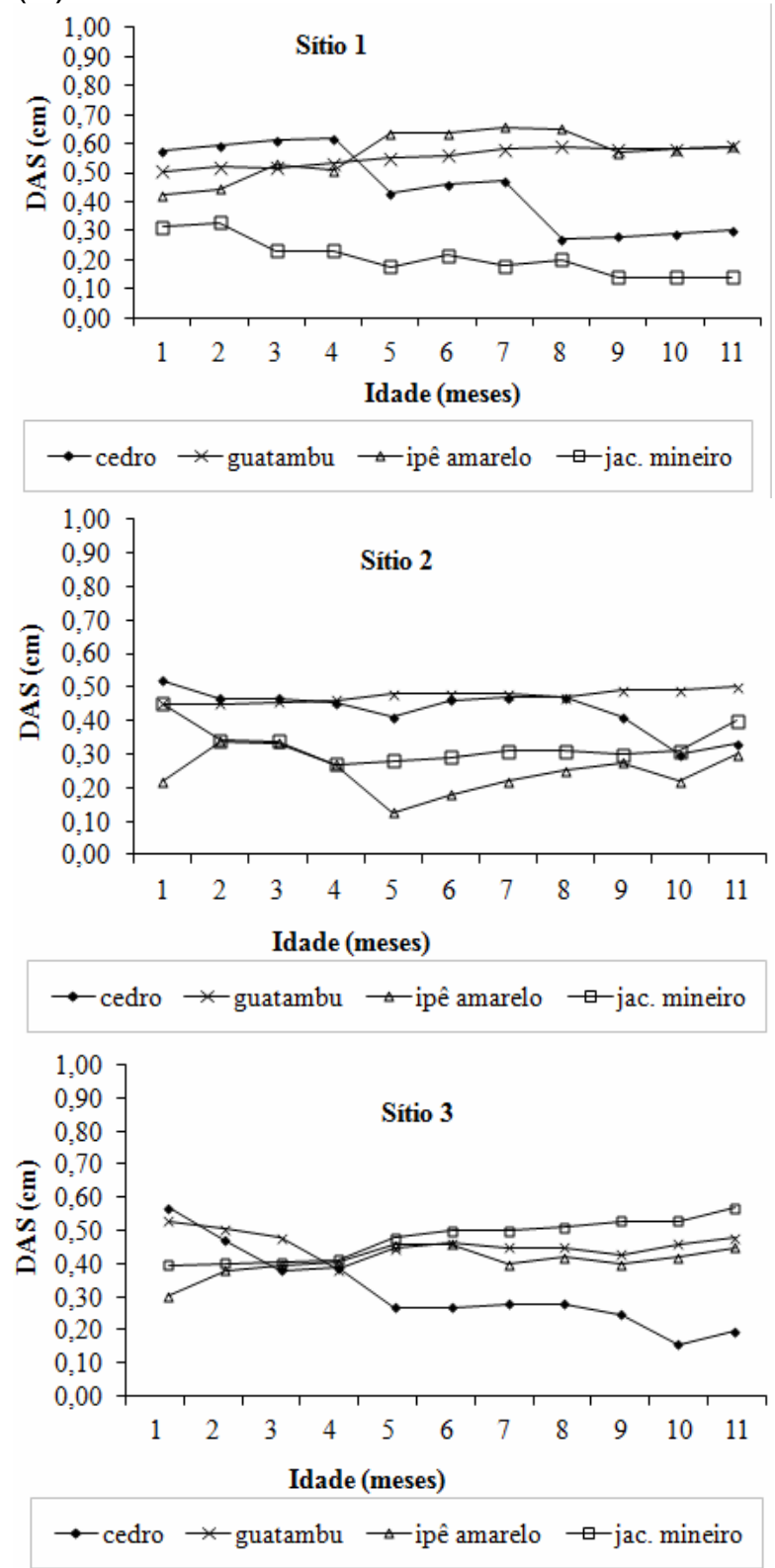

FIGURA 2. Curvas do crescimento médio em diâmetro à altura do solo (DAS) das espécies pioneiras (A) e não pioneiras (B), no sítio 1, sítio 2 e sítio 3 , até 11 meses de idade. Em que: Aroeira $=$ L. molleoides; Mutamba $=G$. ulmifolia; Trema $=T$. micranta; Cedro $=$ C. fissilis; Guatambu $=A$. parvifolium; $H$. serratifolius = Ipê Amarelo e Jac. Mineiro = M. villosum.

Nos sítios 1 e 3, observou-se para as espécies não-pioneiras, tendências distintas de crescimento. No sítio 2 o ritmo de crescimento das espécies foi semelhante, com exceção de $A$. parvifolium, que obteve sempre os maiores valores. Neste sítio, todas as espécies apresentaram as maiores médias de crescimento. Porém, este resultado pode ser considerado inferior, se comparado com estudos realizados no Mato Grosso do Sul, na qual esta espécie obteve altura de $64,4 \mathrm{~cm}$ aos 11 meses (LIMA et al., 2014). Estes autores encontraram uma mortalidade média para esta espécie de $80,5 \%$, ou seja, inferior ao encontrado no presente estudo (100\%). No sítio 3 as espécies, de ambos os grupos, apresentaram ritmos semelhantes de crescimento. 
Com relação ao crescimento em DAS, observa-se em geral (Figura 2), ritmo de crescimento mais rápido entre as pioneiras, o que era esperado para este grupo sucessional. T. micrantha, apresentou menor ritmo de crescimento no sítio 1 e maior nos sítios 2 e 3 . Foi registrado também, menor sobrevivência desta espécie no sítio 1. Possivelmente esse resultado seja consequência do ataque de formigas, uma vez que a espécie é muito atacada em determinadas épocas do ano e quando atacada, apresenta uma redução visível no crescimento, em comparação às plantas não atacadas (MORAES NETO et al., 2010). Entre as espécies não pioneiras, todas apresentaram ritmos semelhantes de crescimento em diâmetro. As espécies que apresentaram menores taxas de crescimento em diâmetro foram as que obtiveram maiores valores de mortalidade, principalmente $C$. fissilis e $M$. villosum.

\section{CONCLUSÃO}

Das sete espécies estudadas, destaca-se $A$. parvifolium, considerando seu crescimento inicial e sua sobrevivência até os 11 meses. De maneira geral, com exceção de $C$. fissilis, todas as espécies demonstram capacidade em se estabelecer em ambientes semelhantes, eliminando-se a necessidade de replantio.

\section{REFERÊNCIAS}

AGUIAR, O.T.; PASTORE, J.A.; ROCHA, F.T.; BAITELLO, J.B. Flora fanerogâmica de um trecho da floresta densa secundária no Parque Estadual da Serra do Mar Núcleo Cunha/Indaiá - Cunha (SP). Revista do Instituto Florestal, São Paulo, v. 13, n. 1, p. 1-18, 2001.

ANDRADE, J.; SANQUETTA, C.R.; UGAYA, C. Identificação de Áreas Prioritárias para Recuperação da Mata Ciliar na UHE Salto Caxias. Espaço Energia, 2005.

ANGELI, A. Cedrella fissilis (Cedro). Identificação de Espécies Florestais: Instituto de Pesquisas e Estudos Florestais. 2015. Disponível em: <http://www.ipef.br/identificacao/cedrella.fissilis.asp>. Acesso em: Março de 2016.

BRASIL. Lei no 12.651, de 25 de Maio de 2012. Diário Oficial da União, Brasília, DF. Disponível em: <http://www.planalto.gov.br/ccivil_03/_ato20112014/2012/lei//12651.htm>. Acesso em: Junho de 2015.

CAMPOS, H. Estatística experimental não-paramétrica. 4.ed. Piracicaba: Esalq, Departamento de Matemática e Estatística, 1983. 349p.

CARVALHO, P.E.R. Espécies florestais brasileiras: recomendações silviculturais, potencialidades e uso da madeira. Colombo, PR, EMBRAPA/CNPF, 1994. 640p.

CASTRO, DILTON. Práticas para restauração da mata ciliar. / organizado por DILTON DE CASTRO; RICARDO SILVA PEREIRA MELLO e GABRIEL COLLARES POESTER. -- Porto Alegre: Catarse - Coletivo de Comunicação, 2012.

CURY, R.T.S. Manual para restauração florestal: florestas de transição / Roberta T. S. Cury, Oswaldo Carvalho Jr. -- Belém: IPAM - Instituto de Pesquisa Ambiental da Amazônia, 2011. -- (Série boas práticas; v. 5) 
DUZ, S.R.; SIMINSKI, A.; SANTOS, M.; PAULILO, M.T.S. Crescimento inicial de três espécies arbóreas da Floresta Atlântica em resposta à variação na quantidade de luz. Revista Brasileira de Botânica, v. 27 n.3, São Paulo. 2004. Disponível em: http://dx.doi.org/10.1590/S0100-84042004000300018

FARIA, R.A.V.B.; BOTELHO, S.A.; SOUZA, L.M. Diagnóstico ambiental de áreas do entorno de 51 nascentes localizadas no município de Lavras, MG. Enciclopédia Biosfera, v. 8, n. 15, p. 648-661, 2012.

FORTES, F.C.A.; RABELO, R.S.; COSTA, I.O.; SILVA, M.M.; SILVA, L.C. IMPACTO AMBIENTAL X AÇÃO ANTRÓPICA: UM ESTUDO DE CASO NO IGARAPÉ GRANDE - BARREIRINHA EM BOA VISTA/RR. In: VI Congresso Brasileiro de Gestão Ambiental, IBEAS - Instituto Brasileiro de Estudos Ambientais. 2015.

LACERDA, D.M.A.; FIGUEIREDO, P.S. Restauração de matas ciliares do rio Mearim no município de Barra do Corda - MA: seleção de espécies e comparação de metodologias de reflorestamento. Acta Amazonica, v.39, n.2, p.295-304, 2009. Disponível em: <http://dx.doi.org/10.1590/S0044-59672009000200008>. doi: 10.1590/S0044-59672009000200008

LIMA, C.T.N.C.; CLEMENTINO, J.A.; LINE, J.D.B.; PIVA, M.A.S.; ROJAS, D.M.; PEREIRA, Z.V. Avaliação e Monitoramento de técnica de restauração ecológica para áreas de preservação permanente do Rio Amambai, município de Amambai - MS. In: 10ํ SINRAD - Simpósio Nacional de Recuperação de Áreas Degradadas, Foz do Iguaçu. 2014.

LORENZI, H. Árvores brasileiras: manual de identificação e cultivo de plantas arbóreas nativas do Brasil. Editora Plantarum. Nova Odessa, SP, 352p., 1992.

MARTELLI, A. Educação ambiental aliada ao método de recuperação por plantio em uma nascente localizada na área urbana do municipio de Itapira - SP. Revista Eletronica em Gestão, Educação e Tecnologia Ambiental - REGET, v.17, n.17, p.3357-3365, 2013. Disponível em: <http://dx.doi.org/10.5902/2236117010889>. doi: $10.5902 / 2236117010889$

MARTINS, S.V. Recuperação de matas ciliares: no contexto do Novo Código Florestal. 3.ed., Viçosa: Aprenda Fácil, v.1, 2014. 220p.

MELO, F.P.; PINTO, S.R.; BRANCALION, P.H.; CASTRO, P.S.; RODRIGUES, R.R.; ARONSON, J.; TABARELLI, M. Priority setting for scaling-up tropical forest restoration projects: Early lessons from the Atlantic Forest Restoration Pact. Environmental Science \& Policy, v. 33, p. 395-404, 2013. Disponível em: <http://www.sciencedirect.com/science/article/pii/S1462901113001494>. doi:10.1016/j.envsci.2013.07.013

MORAES NETO, S.P.; POGGIANI, F.; RODRIGUES, R.R.; CHRISTOFFOLETI, P.J. Efeito do Controle de Gramíneas no Crescimento de Quatro Espécies Arbóreas em Fragmento de Floresta Secundária. 2010. 23p. - (Boletim de pesquisa e desenvolvimento / Embrapa Cerrados, ISSN 1676-918X, ISSN online 2176-509X; 289). 
MORAES, L.F.D.; ASSUMPÇÃO, J.M.; LUCHIARI, C.; PEREIRA, T.S. Plantio de espécies arbóreas nativas para a restauração ecológica na Reserva Biológica de Poço das Antas, Rio de Janeiro, Brasil. Revista Rodriguésia, v.57, n.3, p.477-489, 2006.

OKUYAMA, K.K.; ROCHA, H.C.; NETO, W.H.P.; ALMEIRA, D.; RIBEIRO, D.R.S. Adequação de propriedades rurais ao Código Florestal Brasileiro: Estudo de caso no estado do Paraná. Revista Brasileira de Engenharia Agrícola e Ambiental, v.16, n. 9, p.1015- 1021, 2012. Disponível em: <http://dx.doi.org/10.1590/S141543662012000900013>. doi: 10.1590/S1415-43662012000900013

OLIVA JÚNIOR, E.F. Os impactos ambientais decorrentes da ação antrópica na nascente do Rio Piauí - Riachão do Dantas-SE. Sergipe: Revista Eletrônica da Faculdade José Augusto Vieira, ano V, n.7, 2012.

RUIZ-JAEN, M.C.; AIDE, T.M. Restoration success: How is it being measured? Restoration Ecology, v.13, n.3, p.569-577, 2005. Disponível em: $<$ http://www.globalrestorationnetwork.org/uploads/files/LiteratureAttachments/266_re storation-success---how-is-it-being-measured.pdf>. doi: $10.1111 / \mathrm{j} .1526$ 100X.2005.00072.x

SÁ JÚNIOR, A.; CARVALHO, L.G.; SILVA, F.F.; ALVES, M.C. Application of the Köppen classification for climatic zoning in the state of Minas Gerais, Brazil. Theoretical and Applied Climatology, v. 108, n. 1-2, p. 1-7, 2012. Disponível em:<http://dx.doi.org/10.1007/s00704-011-0507-8>. doi:10.1007/s00704-011-0507-8.

SACRAMENTO, A.S.; SOUZA, D.R.; SANTOS, D.W.F.N. Potencialidades de espécies lenhosas nativas para produção madeireira, cultivadas em solos degradados. Scientia Plena, v.8, n.4, p.1-4, 2012.

SILVA, R.G.; ALVES, M.C.; VILAS BÔAS, R.A.; SARMIENTO, C.M.; BOTELHO, S.A. Monitoramento do processo de Restauração Ecológica de Área de Preservação Permanente por análise espectro-temporal do sensor MODIS. In: XVII SIMPOSIO BRASILEIRO DE SENSORIAMENTO REMOTO - SBSR, João PessoaPB, Brasil. 2015.

SOUZA, L.M.; FARIA, R. A. V. B.; BOTELHO, S. A.; FONTES, M. A. L.; FARIA, J. M. $R$. Potencial da regeneração natural como método de restauração do entorno de nascente perturbada. Revista Cerne, v. 18, n. 4, p. 565-576, 2012. Disponível em: <http://dx.doi.org/10.1590/S0104-77602012000400006>. doi: 10.1590/S010477602012000400006

SOUZA, M.C.; MONTEIRO, R. Levantamento florístico em remanescente de floresta ripária no alto rio Paraná: Mata do Araldo, Porto Rico, Paraná, Brasil. Revista Acta Scientiarum Biological Sciences Maringá, v.7, n.4, p.405-414. 2005. Disponível em: <http://periodicos.uem.br/ojs/index.php/ActaSciBiolSci/article/view/1275>. doi: 10.4025/actascibiolsci.v27i4.1275 